Experimental Research

\title{
Evaluation of the intra-rater variation for the estimation of volume of cerebral structures using the cavalieri principle on magnetic resonance images
}

\author{
Amani Elfaki ${ }^{\mathrm{a}}$, Tahir Osman ${ }^{\mathrm{b}}$, Abdelgani Elsheikh ${ }^{\mathrm{b}}$, Anas Hamdoun ${ }^{\mathrm{c}}$, Bünyamin Şahin ${ }^{\mathrm{a}^{*}}$ \\ ${ }^{a}$ Department of Anatomy, Ondokuz Mayis University, Medical Faculty, Samsun, Turkey \\ ${ }^{b}$ Departments of Anatomy and Psychiatry, Ribat University, MedicalFaculty, Khartoum, Sudan \\ ${ }^{c}$ Department of Radiology, Gazira University, Medical Faculty, Wad Medani, Sudan
}

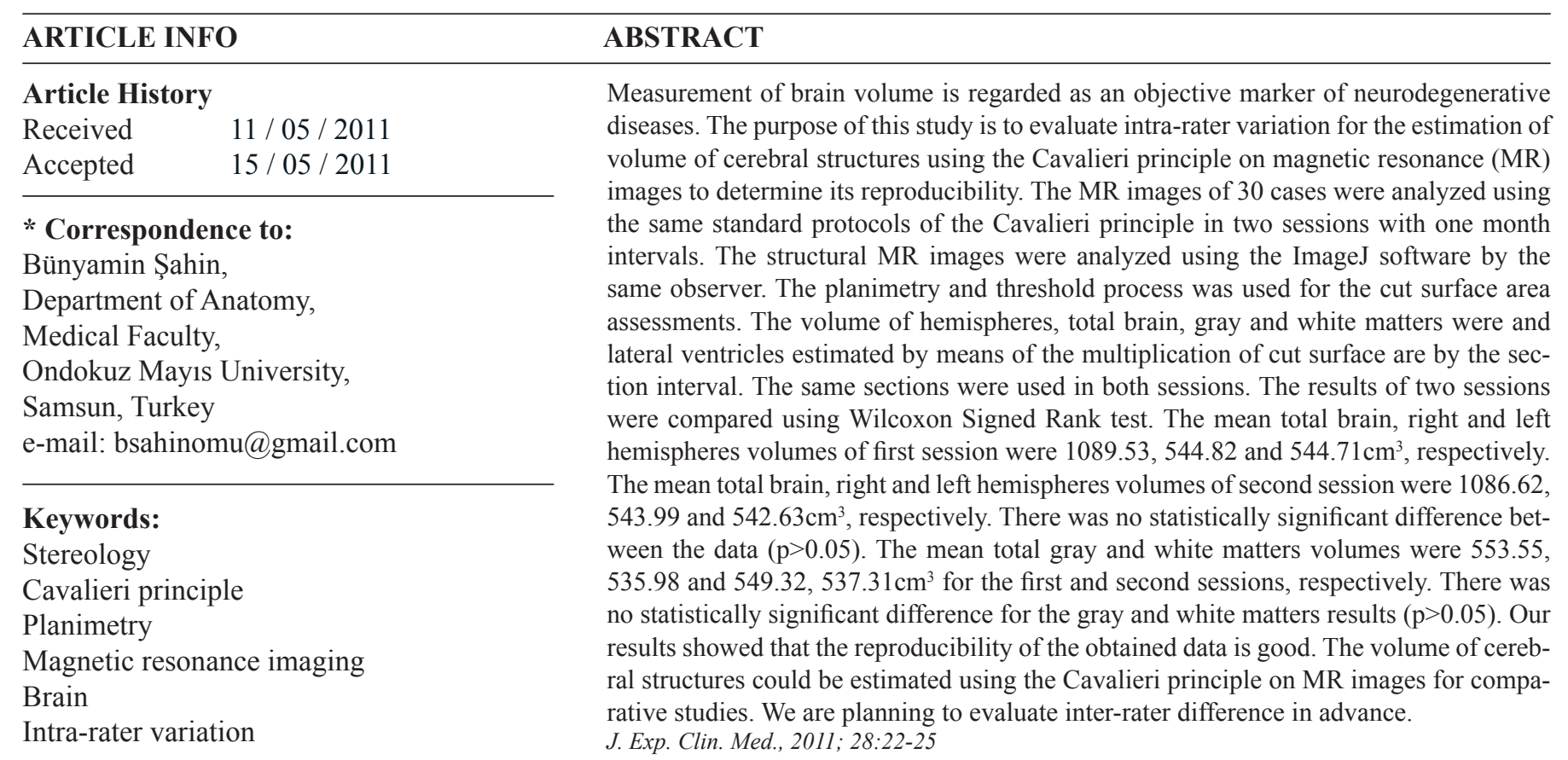

(C) 2011 OMU All rights reserved

\section{Introduction}

Human brain morphometry advanced dramatically by the invention of the modern neuroimaging methods. Magnetic resonance (MR) imaging techniques make it possible to abstract quantifiable information from the slices taken through different parts of the living body. This image slicing approach is directly comparable to the physical or mechanical slicing which is routine in anatomy and pathology both at macroscopic and microscopic levels.

The volume of organs or structures can be obtained using the Cavalieri principle of stereological approaches (Roberts et al., 1993; Cruz-Orive et al., 1997). The requirement for the application of this method is an entire set of two-dimensional slices through the object, provided they are parallel, separated by a known distance, and begins randomly within the object, criteria that are met by standard MR imaging techniques (Roberts et al., 2000; Sahin and Ergur, 2006). In the Cavalieri principle the sum of the sectional surface area of the slices is multiplied by the section thickness and the volume is estimated. The sectional surface area of the slabs could be assessed both the point-counting and planimetry methods of the principle. Planimetry, which involves manually tracing the boundaries of objects of interest on images of sections, is the most commonly used technique for estimation of volume (Gong et al., 1999; Sahin and Ergur, 2006).

There are some studies in the literature evaluating the inter/intra-rater variations of estimates of different organs and structures (Sahin et al., 2003; Odaci et al., 2005). However, we have not seen a study evaluating intra-rater changes of the volume estimates of brain and its components using planimetry technique and MR images.

The purpose of this study is to evaluate intra-rater variation for the estimation of brain volume using the Cavalieri principle on MR images in the living human brain and to determine its reproducibility. 


\section{Experimental Procedure}

We carried out the present study on 30 subjects. They were selected blindly from 88 normal volunteers of the study of the "Quantitative Evaluation of the Cerebral Hemispheres and the Corpus Callosum in Schizophrenic Patients Using Planimetric and Stereological Methods on Magnetic Resonance Images". Subjects age range from 20 to 40. The official permissions are taken from the university and state hospital administrators. All procedures were fully explained to the subjects and written informed consent was obtained. Subjects with head trauma, drug abuse and central neurological disorders were excluded.

Structural MR imaging was done to all subjects in the department of radiology in the National Ribat University/ SUDAN. The scanner is Siemens 1.5 Tesla Magnetom Avanto Vision System. T1-weighted images were obtained using three-dimensional acquisition by Magnetization Prepared Rapid Acquisition (MP-RA); it produces good grey/ white contrast in a very short acquisition time. Slice distance is $1.0 \mathrm{~mm}$, the field of view is $250 \mathrm{read}, 192 \mathrm{~mm}$ phase, $\mathrm{TR}=1657 \mathrm{~ms}, \mathrm{TE}=2.95 \mathrm{~ms}$, band width $180 \mathrm{~Hz} /$ pixel, flip angle $15^{\circ}$, ECHO spacing $=7.5 \mathrm{~ms}$, phase resolution $=100 \%$, slice resolution $=50 \%$, and acquisition time $=5$ minutes $\& 18 \mathrm{sec}-$ onds. The images are in coronal section. This T1-weighted sequence is part of the standard clinical protocol for qualitative and quantitative analysis of the whole brain in patients with epilepsy. The whole procedures take five minutes, without contrast media.

Morphometric measurements were conducted blind to clinical data using ImageJ software. The ImageJ is produced and distributed by the National Institute of Health of USA. The software is in the public domain and was downloaded from the Internet (available at the site: http://rsb.info.nih.gov/ $\mathrm{ij} /$ ). It runs on any computer systems. Measurements from images can be stored separately. This software provides valid and reliable measurements of specific structures using a delineation approach.

The Dicom images of patients were transferred to the software and converted into stack. Systematic random sampling was done since the number of slices in coronal plane about 192 sections. The sampling fraction was $1 / 10$ and $1 / 5$ for the brain and ventricles, respectively. This mean, for example take the 5th section as first than go on 15, 25, 35 for the brain. Therefore, the section interval for the brain was 1 $\mathrm{cm}$ and for the ventricle was $0.5 \mathrm{~cm}$. Finally 15 to 17 sections containing brain images and 14 to 20 sections containing ventricles were obtained.

The midline of the brains and possible bluer borders of the hemispheres were drawn on the images to split them from the surrounding tissues (Fig. 1A). The outer boundaries of the hemisphere were manually delineated (Fig. 1B). Threshold toll of the program was used to delineate the boundaries of the WM then the wand tool of the software was used to delineate the boundaries of hemispheres (Fig. 1C). It was done for the ventricles also (Fig. 1D). The sectional cut surface of the interested structure was measured by the software automatically or semi-automatically. The volume of cerebral hemispheres, ventricles and WM was estimated by the multiplication of total sectional surface area with the section interval (i.e. $1 \mathrm{~cm}$ and $0.5 \mathrm{~cm}$ for the hemisphere and ventricle, respectively) at its shown in the formula below. The total brain volume was estimated by multiplying the volume of both hemispheres and GM volume was obtained by subtracting the volume of white matter (WM) and ventricles from the total hemispheric volume.

$\mathrm{V}=\sum \mathrm{a} \times \mathrm{t}$

Where, $\mathrm{V}$ is the volume, $\sum \mathrm{a}$ is the total sectional area of the structure and $t$ is the interval between the examined sections.

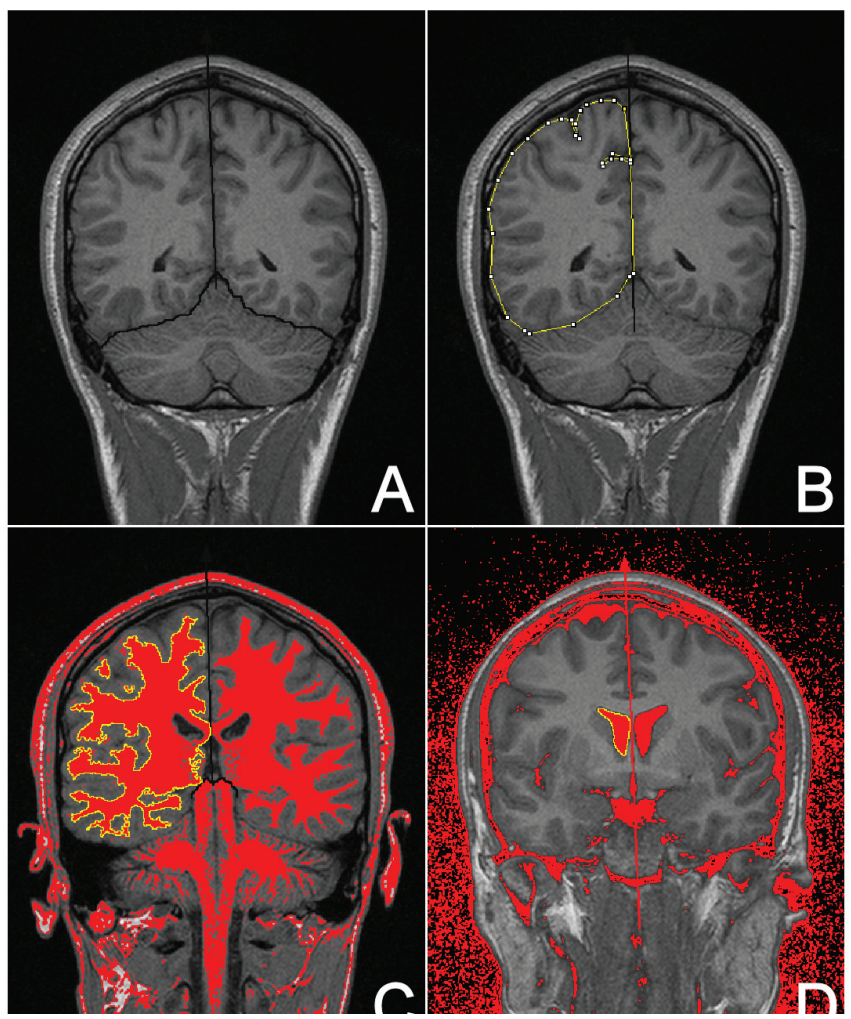

Fig. 1. Image processing in ImageJ for the volume assessment.

A: Delineation of the borders of brain.

$\mathrm{B}$ : Manual planimetry for total brain volume.

C: Tresholding for white matter.

D: Tresholding for lateral ventricles.

Two week later the same procedure was applied and the volume data were obtained by the same observer.

The calculations and the coefficient of error $(\mathrm{CE})$ values were done using Microsoft Excel. A spreadsheet was prepared and the surface area data were transferred from ImageJ to the Excel. Finally, all the calculations were done automatically. Wilcoxon's signed-rank test was applied to compare the results of first and second sessions. Pearson correlation and Bland \& Altman tests were done to see the relation between two session values. A p value equal or less than 0.05 was accepted statistically significant.

\section{Results}

The mean total brain volumes of 30 cases were 1089.5 and $1086.5 \mathrm{~cm}^{3}$ for the first and second sessions, respectively. The mean total WM volumes were 536 and $537.3 \mathrm{~cm}^{3}$, for the first and second sessions, respectively. They were 553.6 and 549.3 $\mathrm{cm}^{3}$ for the total GM volumes in two sessions, respectively (Fig. 2). The values for the total ventricular volumes were same for both sessions, $14.3 \mathrm{~cm}^{3}$. The details of the hemispheres, GM, WM and ventricles were given in the statistical analysis of the data obtained in the first and second sessions 
did not differ from each other $(\mathrm{p}<0.05)$.

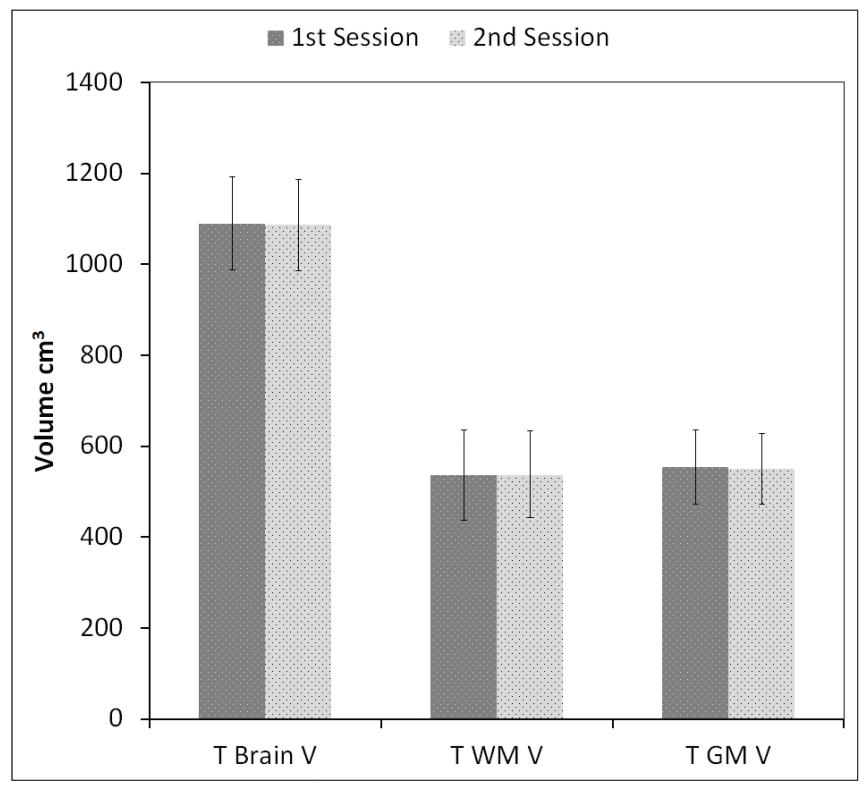

Fig. 2. The mean volumes of total brain (T Brain V), white matter ( T WM V) and gray matter (T GM V) obtained in the first and second sessions in $\mathrm{cm}^{3}$.

Table 1. The volume data of cerebral structures for the first and sec ond sessions. R; right, L; left, T; total, V; volume, Hem; hemisphere, WM; white matter, GM; gray matter, Vent; ventricle.

\begin{tabular}{|lllllll|}
\hline \multicolumn{5}{r}{ Minimum } & \multicolumn{3}{l}{ Maximum } & Mean \\
\cline { 2 - 7 } & 1st & 2nd & 1st & 2nd & 1st & 2nd \\
& Session & Session & Session & Session & Session & Session \\
\hline R Hem V & 440.6 & 446.8 & 665.9 & 660.4 & 544.8 & 544.0 \\
L Hem V & 450.1 & 455.1 & 655.6 & 653.4 & 544.7 & 542.6 \\
T Brain V & 890.7 & 901.9 & 1321.5 & 1313.8 & 1089.5 & 1086.6 \\
R WM V & 184.2 & 180.6 & 393.5 & 383.3 & 277.4 & 278.0 \\
L WM V & 172.9 & 169.4 & 369.8 & 359.9 & 258.6 & 259.3 \\
T WM V & 357.1 & 349.9 & 763.4 & 743.2 & 536.0 & 537.3 \\
R GM V & 193.2 & 199.4 & 373.3 & 365.3 & 267.5 & 266.0 \\
L GM V & 204.2 & 204.4 & 374.3 & 369.8 & 286.1 & 283.3 \\
T GM V & 402.3 & 403.9 & 747.6 & 735.1 & 553.6 & 549.3 \\
R Vent V & 3.4 & 3.7 & 15.0 & 14.9 & 6.8 & 6.8 \\
L Vent V & 3.3 & 3.4 & 12.5 & 13.3 & 7.5 & 7.5 \\
T Vent V & 7.0 & 7.1 & 27.5 & 27.6 & 14.3 & 14.3 \\
\hline
\end{tabular}

The correlation analysis of the estimates showed that there were high correlations between the first and second sessions. The correlation coefficient (r) for the total brain, total WM, total GM and total ventricles were $0.994,0.987,0.979$ and 0.987 , respectively.

We also applied the Bland \& Altman analysis to see the agreement between to session (Fig. 3). There was good agreement between the sessions.

The mean CE of volumes estimates were $0.79,1.43,2.69$ for the hemispheres, WM and ventricles, respectively.

\section{Discussion}

Unbiased and efficient estimation of volume of organs and structures could be done using the Cavalieri principle of the stereological methods with a known precision (Roberts et al., 1993; Cruz-Orive et al., 1997). This method requires sectioning the whole structure with a series of parallel planes. To avoid bias, the first section must be placed at a uniform and random position in a constant interval of length $(\mathrm{t})$ i.e. to start the scanning always at, for example, one $\mathrm{cm}$ from the right

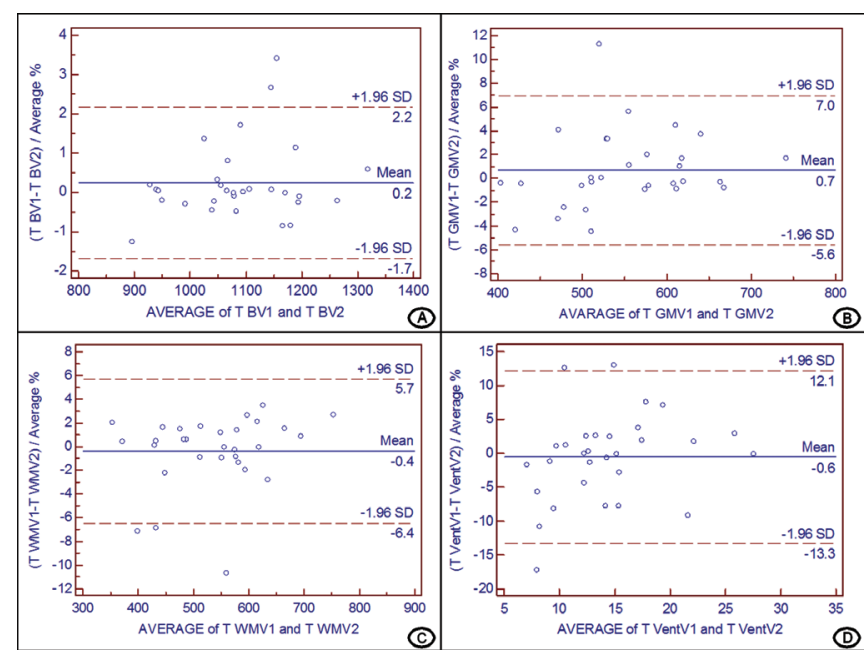

Fig. 3. Results of Blant \& Altman analysis. T; total, V; volume, B; brain, WM; white matter, GM; gray matter, Vent; ventricle.

tip of the object will introduce an unknown amount of bias in general. Moreover, the series of sections must encompass the object entirely. The direction of cutting does not affect the unbiasedness property, but will affect the estimation precision in general (Mackay et al., 1999).

Previous studies showed that the obtained volumes using the Cavalieri principle on sectional radiological images showed little inter-rater variation (Sahin et al., 2003; Odaci et al., 2003; Akbaş et al., 2004). However, these studies are not conducted on the brain or its components.

There are little studies evaluating intra-rater variation of the estimation of brain volume using the MR images. Ronan et al. (2006) reported that the limits of estimates for the volume of brain were felt within \pm 2 SD's of the population and there was a good agreement between repeated measures for the total brain volume data. They used point counting method for the assessment of brain volume in the mentioned study. Bermel et al. (2003) developed a semi-automated measure of brain parenchymal fraction using commercially available edge-finding and thresholding software. Their intra-rater variability was very low and they concluded their software could be used to quantify the brain structures within 30 minutes. We used both the combination of manual delineation and semiautomated planimetry. However, the intra-rater repeatability was similar to the findings of both studies.

Rest of the other available studies evaluates the intrarater variation for the estimation of different part of the brain. Giesel et al. (2009) proposed a new semi-automated method for the estimation of ventricle volume on MR images. Their data revealed that there was great agreement for intra-rater reliability as it is the case for our study. Dawant et al. (1999) also evaluated the volume of brain structure using manual planimetry on MR images. Their findings also showed low intra-rater variability.

The result of point-counting, manual planimetry and semi-automated planimetry there is good agreement for the intra-rater comparisons. Although previous studies used very different strategies for measuring brain volumes in neurodegenerative diseases, they all achieved relatively low intrarater variability, with mean COVs ranging from 0.2 to $2.9 \%$ (Rovaris, 2000).

Beside the intra-rater variation, there are some points which must be regarded for the estimation of brain and its 
structures using the MR images. The section thickness and windowing procedures may contribute bias to the obtained data.

Partial voluming artifact is the result of sectioning and the thickness of sections in MR imaging. The finite resolution of images is recognized as an important problem of the MR imaging technique. The intensity of signal cannot be separated within each voxel when it arises from different tissue compartments and this produces what is referred to as partial voluming artifact. García-Fiñana et al. (2003) recommends that the relatively high resolution is not sufficient to solve the tissue boundaries ambiguity. To fix the contribution of effect of section thickness standard section thickness should be used for the research studies (Sahin and Ergur, 2006).

Windowing is an adjustment for the best visualization of frames of MR images. During the windowing of frames, different levels of settings to obtain best view are chosen. Windowing adjustments are related to the nature of scanned structure and the imaging technique. Moreover, Diederichs et al. (1996) showed that a proper windowing must be chosen to obtain maximum intensity projections. In the living sub- jects, the skull is filled up with brain, meninges and cerebrospinal fluid. During the scanning all those structures absorbs or reflects magnetic resonance signals in different degrees. Standard windowing parameters may eliminate the effect of windowing for the estimation of volumes of structures.

As a conclusion, we may say that the estimation of volume of brain and its structures do not affected by the applicant when using the manual or semi-automated planimetry techniques. Therefore, the studies having data obtained by the same observer may have reliable and acceptable data. However, inter-rater differences and the other factor such as the section thickness, the number of sections used in the study and the windowing procedures must be taken in to account.

\section{Acknowledgements:}

The authors thank to the technicians of the department of radiology in the National Ribat University/ SUDAN, for their great contribution to this study and to the subjects who participated in collection of data. This study was supported by the Organization for Women in Science for the Developing World (OWSDW).

\section{REFERENCES}

Akbas, H., Sahin, B., Eroglu, L., Odaci, E., Bilgic, S., Kaplan, S., Uzun, A., Ergur, H , Bek, Y. 2004. Estimation of breast prosthesis volume by the Cavalieri principle using magnetic resonance images. Aesthetic. Plast. Surg. 28, 275-280.

Bermel, R.A., Sharma, J., Tjoa, C.W., Puli, S.R., Bakshi, R. 2003. A semiautomated measure of whole-brain atrophy in multiple sclerosis. J. Neurol. Sci. 208, 57-65.

Cruz-Orive, L.M. 1997. Stereology of single objects. J. Microsc. 186, 93-107.

Dawant, B.M., Hartmann, S.L., Thirion, J.P., Maes, F., Vandermeulen, D., Demaerel P. 1999. Automatic 3-D segmentation of internal structures of the head in MR images using a combination of similarity and free-form transformations, Part I, Methodology and validation on normal subjects. IEEE Trans Med Imaging. 18, 909-916.

Diederichs, C.G., Keating, D.P., Glatting, G., Oestmann, J.W. 1996. Blurring of vessels in spiral CT angiography: effects of collimation width, pitch, viewing plane, and windowing in maximum intensity projection. J. Comput. Assist. Tomogr. 20, 965-974.

García-Fiñana, M., Cruz-Orive, L.M., Mackay, C., Pakkenberg, B., Roberts, N. 2003. Comparison of MR imaging against physical sectioning to estimate the volume of human cerebral compartments. NeuroImage. 18, 505-516.

Giesel, F.L., Hart, A.R., Hahn, H.K., Wignall, E., Rengier, F., Talanow, R., Wilkinson, I.D., Zechmann, C.M., Weber, M.A., Kauczor, H.U., Essig, M., Griffiths, P.D. 2009. 3D reconstructions of the cerebral ventricles and volume quantification in children with brain malforma tions. Acad Radiol. 16, 610-617.

Gong, Q.Y., Tan, L.T., Romaniuk, C.S., Jones, B., Brunt, J.N., Roberts N. 1999. Determination of tumour regression rates during radiotherapy for cervical carcinoma by serial MRI, comparison of two measurement techniques and examination of intraobserver and interobserver variability. Br. J. Radiol. 72, 62-72.

Mackay, C.E., Pakkenberg, B., Roberts, N. 1999. Comparison of compartment volumes estimated from MR images and Physical sections of formalin fixed cerebral hemispheres. Acta. Stereol. 18, 149-159.

Odaci, E., Sahin, B., Sonmez, O.F., Kaplan, S., Bas, O., Bilgic, S., Bek, Y., Ergür, H. 2003. Rapid estimation of the vertebral body volume, a combination of the Cavalieri principle and computed tomography images. Eur. J. Radiol. 48, 316-326.

Roberts, N., Cruz-Orive, L.M., Reid, N.M., Brodie, D.A., Bourne, M., Edwards, R.H. 1993. Unbiased estimation of human body composition by the Cavalieri method using magnetic resonance imaging. J. Microsc. 171, 239-253.

Roberts, N., Puddephat, M.J., McNulty, V. 2000. The benefit of stereology for quantitative radiology. Br. J. Radiol. 73, 679-697.

Ronan, L., Doherty, C.P., Delanty, N., Thornton, J., Fitzsimons, M. 2006. Quantitative MRI, a reliable protocol for measurement of cerebral gyrification using stereology. Magn. Reson. Imaging. 24, 265-272.

Rovaris, M. 2000. Sensitivity and reproducibility of volume change measurements of different brain portions on MR scans from patients with multiple sclerosis. J. Neurol. 247, 960- 965.

Sahin, B., Emirzeoglu, M., Uzun, A., Incesu, L., Bek, Y., Bilgic, S., Kaplan S. 2003. Unbiased estimation of the liver volume by the Cavalieri principle using magnetic resonance images. Eur. J. Radiol. 47, 164-170.

Sahin, B., Ergur, H. 2006. Assessment of the optimum section thickness for the estimation of liver volume using magnetic resonance images, a stereological gold standard study. Eur. J. Radiol. 57, 96-101. 